\title{
Conflictos semióticos y niveles de algebrización en aspirantes a Ingeniería
}

\section{Semiotic conflicts and levels of algebrization in engineering candidates}

\author{
Víctor Larios Osorio ${ }^{1}$ \\ Patricia Isabel Spíndola Yáñez ${ }^{2}$ \\ Omar Cuevas Salazar ${ }^{3}$ \\ Jesús Jerónimo Castro ${ }^{4}$
}

\begin{abstract}
Resumen: Este trabajo presenta un estudio sobre el desarrollo del conocimiento algebraico de aspirantes a las carreras de Ingeniería de la Universidad Autónoma de Querétaro, México. Se aplicó un instrumento con ítems abiertos a los aspirantes del ciclo 2017-2018 y de contenido de matemático de bachillerato. En el análisis de las respuestas se identificaron prácticas matemáticas, significados personales y conflictos semióticos relacionados con niveles de algebrización que no son apropiados para un desarrollo adecuado de futuros ingenieros. Esto permitirá proponer cambios en el diseño del proceso de admisión, en la parte curricular de las carreras existentes y en el diseño de acciones que puedan fortalecer y apoyar la formación académica de los aspirantes una vez que hayan sido aceptados.
\end{abstract}

Fecha de recepción: 10 de diciembre de 2018. Fecha de aceptación: 4 de junio de 2021.

1 Universidad Autónoma de Querétaro, Facultad de Ingeniería, División de Investigación y Posgrado, vil@uaq.mx, orcid.org/0000-0002-4454-8516

2 Universidad Autónoma de Querétaro, Facultad de Ingeniería, División de Investigación y Posgrado, spindola@uaq.mx

3 Instituto Tecnológico de Sonora, Departamento de Matemáticas, ocuevas@itson.edu.mx, orcid.org/00000003-0113-0475

${ }^{4}$ Universidad Autónoma de Querétaro, Facultad de Ingeniería, jesus.jeronimo@uaq.mx, orcid.org/00000002-6601-0004 
Palabras clave: Formación de ingenieros, Conflictos semióticos, Niveles de algebrización.

\begin{abstract}
This paper shows a study on the level of development of algebraic knowledge of applicants to engineering careers of the Autonomous University of Querétaro, México. An instrument with open items was applied to applicants for the 2017-2018 cycle and for high school mathematics content. In the analysis of the responses we identified mathematical practices, personal meanings and semiotic conflicts related to levels of algebrization that are not appropriate for the adequate development of future engineers. This will make possible to propose changes in the design of the admission process, in the curricular part of existing careers and in the design of actions that can strengthen and support the academic training of applicants once they have been accepted.
\end{abstract}

Keywords: Engineer training, Semiotic conflicts, Levels of algebrization.

\title{
INTRODUCCIÓN Y ANTECEDENTES
}

La Universidad Autónoma de Querétaro (UAQ) en su Facultad de Ingeniería oferta doce carreras universitarias, de las cuales nueve corresponden a diversas ingenierías (Civil, Automatización, Industrial y Manufactura, Electromecánica, Mecánica y Automotriz, Nanotecnología, Física, Agroindustrial y Biomédica). Estas carreras tienen un tronco común de dos años que incluyen cursos de las áreas de matemáticas, física y química, principalmente. Los cursos del área de matemáticas incluyen algebra lineal, probabilidad y estadística, cálculo (diferencial, integral y multivariable) y ecuaciones diferenciales.

Con estos requisitos, para la primera parte de las carreras se planteó desde hace unos años un proceso de admisión que incluye un curso propedéutico y la aplicación del Examen de Competencias Básicas (Excoba). El curso está orientado a proporcionar a los aspirantes un bagaje general y homogéneo en algunas áreas del bachillerato (matemáticas, física y química), y además cuenta con un proceso de evaluación continua a través de las sesiones, tareas y algunos exámenes. El Excoba, por su parte, se orienta a la evaluación a través de una herramienta estandarizada. Al final ambos componentes se ponderan y se obtiene un puntaje con el que se determina el ingreso de cada uno de los aspirantes. 
Parte del interés institucional es determinar la situación académica en la que arriban los aspirantes y así proponer adecuaciones en el proceso, ya que se tienen datos preocupantes como el hecho de que entre 2012 y 2018 solo 22\% de los aspirantes han sido admitidos. ${ }^{5}$ Por otro lado, existe la tendencia de migrar el proceso de admisión de una modalidad presencial a una semipresencial, por lo que se están realizando propuestas académicas en ese sentido.

Este proceso de ingreso a la Facultad es influido por la transición del bachiIlerato a la universidad que, a diferencia de algunos de los niveles previos, no tiene una articulación oficial y depende de cada institución y de las condiciones particulares de cada alumno. Larrazolo et al. (2013), por ejemplo, llevaron a cabo un análisis de los resultados de una prueba estandarizada diseñada para evaluar competencias matemáticas básicas ${ }^{6}$ y mostraron "que los estudiantes que aspiran a ingresar al nivel de educación superior [en cinco entidades federativas] presentan serias deficiencias en los aprendizajes esperados de matemáticas de los niveles de primaria y de secundaria" (p. 1157). Señalan que algunas de las áreas importantes en estas deficiencias están en la aritmética y el álgebra. Comentarios similares exponen Cuevas et al. (2018) al analizar dos cohortes de ingreso en el Instituto Tecnológico de Sonora en aritmética, álgebra, geometría analítica y nociones básicas de cálculo.

Ahora bien, para abordar el estudio del cálculo de manera exitosa es necesario que los alumnos manejen conceptos y algoritmos algebraicos de manera apropiada que les permita ocuparse del aprendizaje de conceptos nuevos, ligados a la modelación del movimiento, y no se detengan en resolver situaciones algebraicas. Sin embargo, en varios trabajos previos dentro de la misma UAQ se ha mostrado que los alumnos de bachillerato prefieren utilizar estrategias aritméticas para plantear y resolver problemas, además de aceptar generalizaciones a partir de algunos casos particulares (Larios, 2018; Larios et al., 2017; Martínez et al., 2015). En este sentido el interés en considerar este aspecto en el desarrollo académico de los estudiantes que inician la formación específica de ingenieros toma relevancia.

Kieran (2003), por su parte, clasifica las tareas algebraicas escolares en tres tipos:

${ }^{5}$ Se hace la aclaración de que en la Facultad la cantidad de admitidos no está basada en un cupo máximo, sino en un puntaje mínimo fijo.

${ }^{6}$ Por la fecha del análisis la prueba estandarizada referida era el Examen de Habilidades y Conocimientos Básicos (Exhcoba). 
- Las generacionales, que incluyen la producción de expresiones o ecuaciones como objetos algebraicos.

- Las transformacionales, que son actividades que se basan en el manejo de las reglas como, por ejemplo, la simplificación de términos, la factorización, el resolver ecuaciones, simplificar expresiones, etcétera.

- Las actividades matemáticas globales, meta-nivel, en las cuales el Álgebra se utiliza como una herramienta aunque no son exclusivas a esta, como es el caso de la modelación, la resolución de problemas, las demostraciones, etcétera.

Sin embargo, y pesar de que es común que durante los años previos a los estudios universitarios (y al bachillerato) los estudiantes se enfrenten a los tres tipos de actividades en diferentes momentos de su paso por la escuela, en la literatura se encuentran referencias sobre las dificultades en su aprendizaje por diversas razones como, por ejemplo, la forma en que se aborda el aprendizaje de la aritmética en la escuela primaria y su transición hacia la secundaria (Kieran, 2003), aspectos vinculados con la polisemia de las variables (Rojano, 2006), el uso del signo de igual (Frost, 2015), entre otros.

Por su parte, Kieran (2003, p. 130) considera que "la resolución de ecuaciones y la simplificación de expresiones requieren la habilidad de razonar acerca de las operaciones". Esto quiere decir que se requiere que la manipulación algebraica que se utiliza como herramienta para el estudio de las matemáticas en ingeniería no sea automática y sin sentido, sino soportada por una reflexión. No obstante, se ha mostrado desde hace varias décadas que cuando los alumnos no logran ese nivel de reflexión entonces tienden a recurrir a la memorización de reglas y procedimientos, convenciéndose que de que las matemáticas se basan en reglas o "recetas" (NMAP, 2008).

Con estas consideraciones se ha planteado un proyecto que, entre otros aspectos, pretende determinar cuál es el nivel de desarrollo del conocimiento algebraico de los aspirantes a las carreras de Ingenierías en la Facultad de Ingeniería de la UAQ, así como los principales obstáculos que se constituyen en dificultades para su futuro desarrollo académico. Esto permitirá proponer cambios en el diseño del proceso de admisión, en la parte curricular de las carreras existentes y en el diseño de acciones que puedan fortalecer y apoyar la formación académica de los aspirantes una vez que hayan sido aceptados. 


\section{MARCO TEÓRICO}

Para el análisis de la información se considerará el Enfoque Ontosemiótico para la Cognición y la Instrucción Matemáticas (EOS) (Godino et al., 2007) que toma en cuenta los objetos matemáticos, su naturaleza y formas de expresión para analizar las prácticas matemáticas considerando algunas herramientas metodológicas.

En particular tomaremos en cuenta, por un lado, las funciones semióticas (Godino y Batanero, 2003) como un medio para identificar conflictos semióticos expresados por los alumnos. Por otro lado, se tomará en cuenta un modelo del pensamiento algebraico en el que se distinguen niveles de razonamiento algebraico (Godino et al., 2014; Godino et al., 2015) para identificar la situación de los alumnos aspirantes a la Facultad.

\section{LAS FUNCIONES Y LOS CONFLICTOS SEMIÓTICOS}

Si bien se acepta, generalmente, que los objetos matemáticos tienen un significado consensuado en la comunidad matemática -el denominado significado institucional-, ocurre que es común que este no coincida con el significado construido por los estudiantes que están aprendiendo el denominado significado personal. Hacer esta distinción desde el EOS tiene sentido porque, en el campo educativo identificar estas diferencias permite establecer el desarrollo del individuo que aprende y además de plantear estrategias tanto en el salón de clase como a nivel de propuestas curriculares.

En este sentido la noción de función semiótica se constituye en un instrumento metodológico útil para un análisis el cual, como mencionan Aznar et al. (2016), permita:

- "Identificar los conflictos de significados que pueden obstaculizar el aprendizaje en un proceso de instrucción.

- "Obtener información relevante para el diseño de estrategias de enseñanza" (p. 672).

Godino y Batanero (2003), al igual que Hjelmslev (1971) y Eco (2005), conciben la función semiótica como una correspondencia que pone en juego tres componentes: 
- Un plano de expresión, que le corresponde al signo.

- Un plano de contenido, que le corresponde al significado del signo.

- Un criterio, que le corresponde a un código o regla que relaciona los planos anteriores.

Así, cuando se utilizan los signos y se les otorga un significado se establece una correlación que está influida por el contexto y que no actúan independientemente. Como dice Hjemslev (1971, p. 74), "no puede concebirse una función sin sus terminales, y los terminales son únicamente puntos finales de la función y, por tanto, inconcebibles sin ella". En palabras de Eco (2005, p. 84), "hablando con propiedad, no existen signos, sino funciones semióticas".

Así, como mencionan Aznar y sus colegas (2016), podemos determinar o modelar los significados personales establecidos a los objetos matemáticos a través del análisis de las funciones semióticas involucradas, a fin de contrastarlas con aquellas que representan los significados institucionales o pretendidos.

En este sentido cuando el individuo establece una función semiótica para un objeto que le conduce a un uso diferente al que se tiene de referencia y que, genera contradicciones, entonces se dice que existe un conflicto. Ahora bien, el conflicto se denomina como semiótico cuando existe una discordancia entre el significado, es decir la interpretación, que se le da a una misma expresión o representación por parte de dos sujetos que bien pueden ser individuos o comunidades (instituciones).

Como lo plantean Godino et al. (2007), cuando la disparidad de significados se produce entre instituciones se habla de un conflicto semiótico epistémico. Y si la discordancia aparece en las prácticas matemáticas de un mismo sujeto se denomina conflicto semiótico cognitivo.

Como se verá más adelante, esta idea nos ha permitido identificar dificultades en el aprendizaje que han realizado los participantes durante su desarrollo académico.

\section{Niveles de algebrización}

Godino, Aké y colaboradores (2014) han propuesto un modelo de pensamiento algebraico en el que se distinguieron inicialmente un nivel de razonamiento puramente aritmético, dos de actividad protoalgebraica y un tercero de actividad 
claramente algebrizada. En un trabajo posterior ampliaron el modelo para incluir finalmente seis niveles de algebrización (Godino et al., 2015).

Es importante mencionar lo que se entiende por niveles de algebrización:

Los niveles de algebrización se asignan a la actividad matemática que realiza el sujeto que resuelve un problema o tarea matemática, no a las propias tareas, las cuales se pueden resolver de distintas maneras, pudiendo poner en juego una actividad algebraica diferente. (Godino et al., 2015, p. 119)

Además, los niveles planteados son los siguientes (Godino et al, 2015):

Nivel 0 (aritmético): Se opera con objetos intensivos y utilizando registros de representación de lenguaje natural, numérico, icónico, gestual.

Nivel 1: En tareas estructurales, pueden intervenir datos desconocidos y aplicarse relaciones y propiedades de las operaciones. En tareas funcionales, se reconocen los objetos intensivos y se calcula con objetos extensivos. La igualdad se usa como equivalencia y se utilizan como lenguajes el natural, numérico, icónico, gestual.

Nivel 2: Se utilizan representaciones simbólicas (literales) para referirse a objetos intensivos reconocidos, pero vinculados a la información especial, temporal y el contexto. Aunque se reconocen la generalidad, no se opera con variables para obtener formas canónicas.

Nivel 3: Aparecen variables o indeterminadas que se manejan de manera analítica, con un lenguaje simbólico y sin hacer referencia a la información contextual.

Nivel 4: Se utilizan parámetros y coeficientes variables que permiten el estudio de familias de ecuaciones y funciones.

Nivel 5: Se hace uso de más de un parámetro, junto con variables o cantidades indeterminadas, para realizar cálculos analíticos con base en reglas sintácticas.

Nivel 6: El estudio de las estructuras algebraicas en sí se lleva a cabo a partir de definiciones y propiedades como estructura algebraica.

Es importante mencionar que, por un lado, estos autores consideran que es posible la existencia de niveles más avanzados propios de estudios universitarios en matemáticas (Godino et al., 2015). Por otro, y como ya se mencionó al inicio de la sección, los primeros tres niveles (0-2) corresponden a prácticas matemáticas aritméticas protoalgebraicas, por lo que los niveles de prácticas algebraicas propiamente dichas son a partir del nivel 3.

Al aprovechar estas herramientas abordaremos el análisis realizado que es expuesto más adelante. 


\section{MÉTODO}

\section{Población}

La población con la que se trabajó constó de 195 aspirantes a las carreras de Ingeniería de la Facultad de Ingienería, quienes provenían del nivel medio superior (bachillerato) tanto de escuelas locales (incluidos los planteles de la Escuela de Bachilleres de la misma institución) como de escuelas foráneas.

La aplicación se realizó al inicio del proceso de admisión a fin de determinar las características de los posibles futuros alumnos.

\section{Instrumento}

Para el diagnóstico inicial de los aspirantes se desarrolló un instrumento con cinco ítems, con un contenido centrado en álgebra, geometría analítica y precálculo, aunque para el objetivo de este trabajo solo se consideraron tres ítems, que a continuación se describen.

\section{Ítem 1}

Es de tipo transformacional, pues se requiere la manipulación algebraica de una expresión:

En la siguiente expresión despeja la variable $R$. Escribe todos los pasos que utilizaste.

$$
L=\frac{1}{w} \sqrt{Z^{2}-R^{2}}
$$


En el proceso esperado de un alumno para resolverlo se involucra la observación de las siguientes restricciones para las variables:

i. $\quad w \neq 0$,

ii. $\quad w L \geq 0$, es decir, o bien $L=0$, o bien $L y$ w tienen el mismo signo, $y$

iii. $\quad$ si $Z^{2}-R^{2} \geq 0$.

Así, con estas consideraciones, la manipulación algebraica quedaría como sigue:

$$
\begin{aligned}
L & =\frac{1}{w} \sqrt{Z^{2}-R^{2}}, \\
w L & =\sqrt{Z^{2}-R^{2}}, \\
(w L)^{2} & =\left(\sqrt{Z^{2}-R^{2}}\right)^{2}, \\
(w L)^{2} & =Z^{2}-R^{2}, \\
R^{2} & =Z^{2}-(w L)^{2}, \\
R^{2} & =Z^{2}-w^{2} L^{2}, \\
R & = \pm \sqrt{Z^{2}-w^{2} L^{2}} .
\end{aligned}
$$

Una manera de representar gráficamente los objetos matemáticos primarios involucrados (definiciones, conceptos, propiedades, proposiciones, procedimientos, argumentos, lenguaje), así como sus relaciones, es a través de la denominada configuración epistémica (ver llustración 1). 


\section{Situación:}

Es la planteada en el ítem y que se refiere a la manipulación sintáctica de símbolos algebraicos.

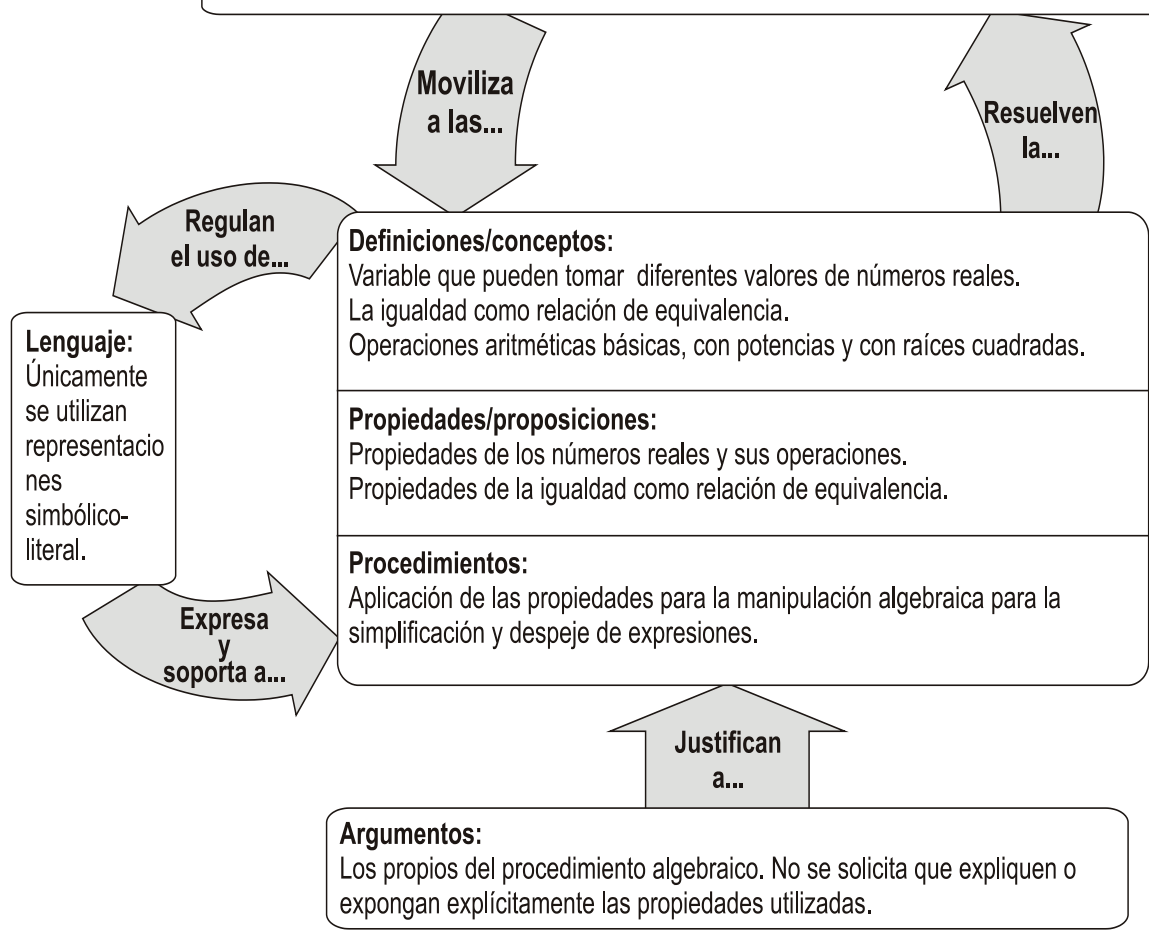

Ilustración 1. Configuración epistémica del ítem 1.

Así pues, se tiene que la situación planteada en el ítem moviliza una serie de definiciones, conceptos, proporciones y procedimientos a fin de resolver, precisamente, dicha situación. Esta movilización de objetos primarios se manifiestan por medio de lenguajes (que se pueden expresar en diversos registros de representación semiótica) que, además, son regulados precisamente por las definiciones, las propiedades, etcétera, y que contienen argumentos que justifican a esos objetos.

Aunque parezca que el interés es simplemente determinar si el alumno es capaz de realizar un despeje, al pedirles que expongan todos los pasos es posible identificar el uso de los símbolos algebraicos como variables genéricas, así como la manipulación sintáctica a través de la aplicación de propiedades de los números reales, sus operaciones y de la igualdad como relación de equivalencia. 
Además, se buscó identificar el uso que le dan a estas propiedades con la intención de determinar qué tan necesario es reforzar aspectos conceptuales y procedimentales del álgebra desde la Secundaria y que se abordan explícitamente en los cursos de la Escuela de Bachilleres de nuestra Universidad.

\section{Ítem 2}

A Baltazar le regalaron un juego de cubos. El chico trata de acomodar todos los cubos para formar un cuadrado, pero descubre que le faltan siete cubos. Entonces intenta hacer un cuadrado cuyo lado es 1 cubo más chico que el anterior, pero entonces le sobran 10 cubos. ¿Cuántos cubos le regalaron a Baltazar?

Una solución posible, en el ámbito del Álgebra, es la siguiente:

Si se toma a $n$ como la cantidad de cubos que le dieron a Baltazar y si $k$ es la cantidad de cubos que tendría el primer cuadrado que intenta formar, entonces tenemos las siguientes expresiones que corresponden a cada uno de los dos cuadrados que se intentan formar:

$$
\begin{aligned}
& n+7=k^{2}, \\
& n-10=(k-1)^{2} .
\end{aligned}
$$

Así que se tendría que resolver el sistema de ecuaciones que le corresponden:

$$
\left\{\begin{array} { l } 
{ n + 7 = k ^ { 2 } } \\
{ n - 1 0 = ( k - 1 ) ^ { 2 } }
\end{array} \Leftrightarrow \left\{\begin{array} { l } 
{ n = k ^ { 2 } - 7 } \\
{ n = k ^ { 2 } - 2 k + 1 + 1 0 }
\end{array} \Leftrightarrow \left\{\begin{array}{l}
n=k^{2}-7 \\
n=k^{2}-2 k+11
\end{array}\right.\right.\right.
$$

Al utilizar, por ejemplo, el método de igualación se obtienen las expresiones:

$$
\begin{gathered}
k^{2}-7=k^{2}-2 k+11, \\
-7=-2 k+11, \\
2 k=18, \\
k=\frac{18}{2}=9 .
\end{gathered}
$$

Al sustituir en una de las primeras expresiones obtenemos el valor buscado: $n=74$. 
Este ítem es una tarea de tipo generacional (Kieran, 2003), pues se le pide a los participantes que planteen ecuaciones que representen situaciones cuantitativas a partir patrones geométricos.

El proceso de resolución que utilicen los participantes ayuda a identificar en términos procedimientales el nivel de algebrización y el tipo de argumentación utilizada, porque se hacen explícitos el tipo de objetos (intensivos o extensivos) con que se opera. El proceso de resolución propuesto como ideal involucra a variables que pueden manejarse con un lenguaje simbólico. Así que los procedimientos propuestos por los participantes, con el tipo de objetos (intensivos o extensivos) con que se opera, los métodos a los que hagan referencia (análiticos, por ejemplo) o el tipo de lenguaje que se utilice, permite identifical el nivel de algebrización correspondiente.

Los objetos primarios involucrados, junto con sus relaciones, se representan gráficamente en la llustración 2.

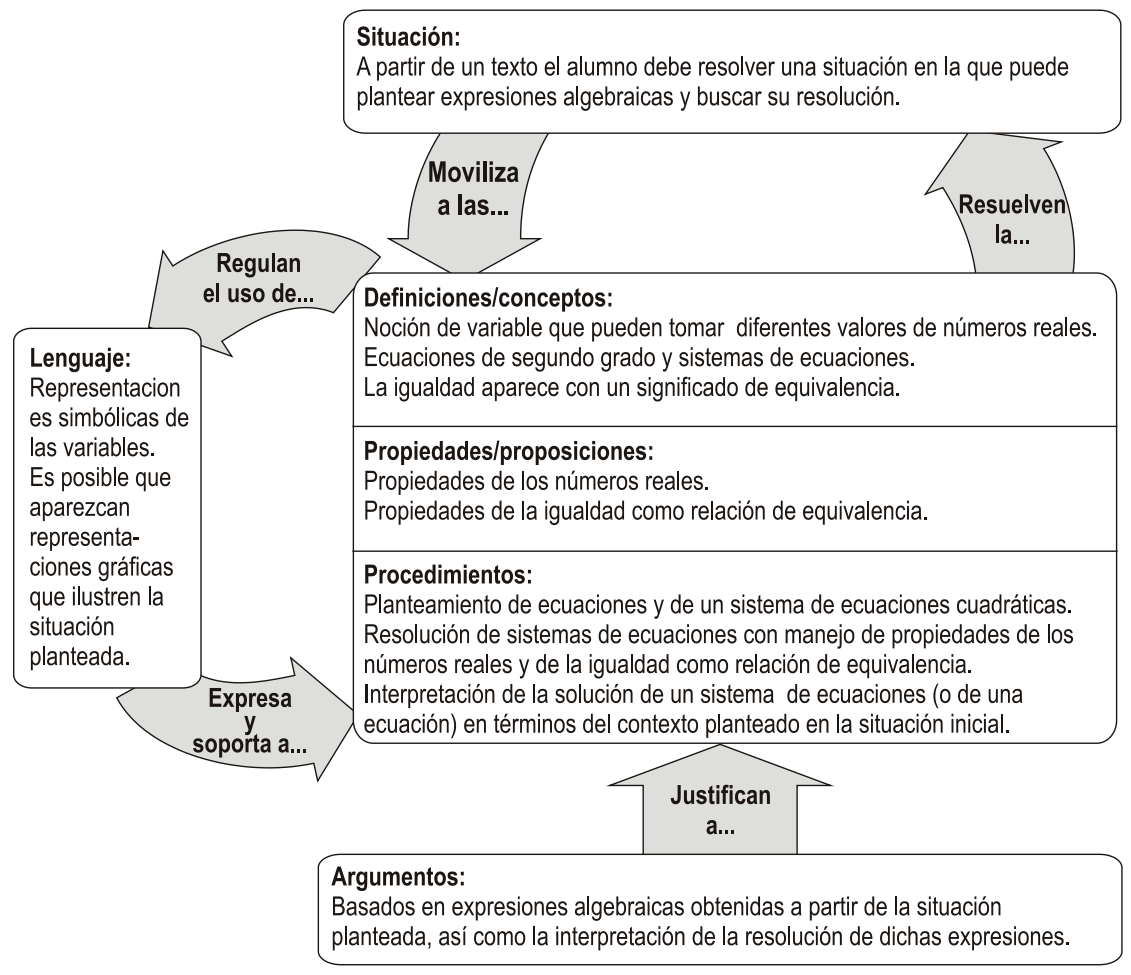

Ilustración 2. Configuración epistémica del ítem 2. 


\section{Ítem 3} Si a es un número positivo, b y c son números negativos, ċla expresión $\frac{(b+c)^{3}(a-3)^{2}}{b^{2} c^{3}}$ tiene
un valor positivo o negativo? Justifica tu respuesta lo más que puedas.

Se ha considerado que este ítem es una actividad matemática global meta-nivel, pues requiere de una manipulación algebraica (actividad transformacional) pero orientada a la identificación de propiedades de los números reales para presentar una justificación ordenada.

Así que se espera que, los participantes afirmen que la expresión tiene un valor positivo y lo justifiquen de una manera similar a la siguiente:

i. Si $b<0$ y $c<0$, entonces $b+c<0$, por lo que $(b+c)^{3}<0$.

ii. Sin importar el valor de $a$, se cumple que $(a-3)^{2}>0$.

iii. Así que $(b+c)^{3}(a-3)^{2}<0$.

iv. Por las condiciones expuestas en $i$ ), $b^{2}>0$ y $c^{3}<0$, por lo que $b^{2} c^{3}<0$. v. Así que, por las condiciones de iii) y iv) se tiene que $\frac{(b+c)^{3}(a-3)^{2}}{b^{2} c^{3}}>0$.

Los objetos y procesos, así como sus representaciones se muestran a continuación en la forma de una configuración epistémica: 


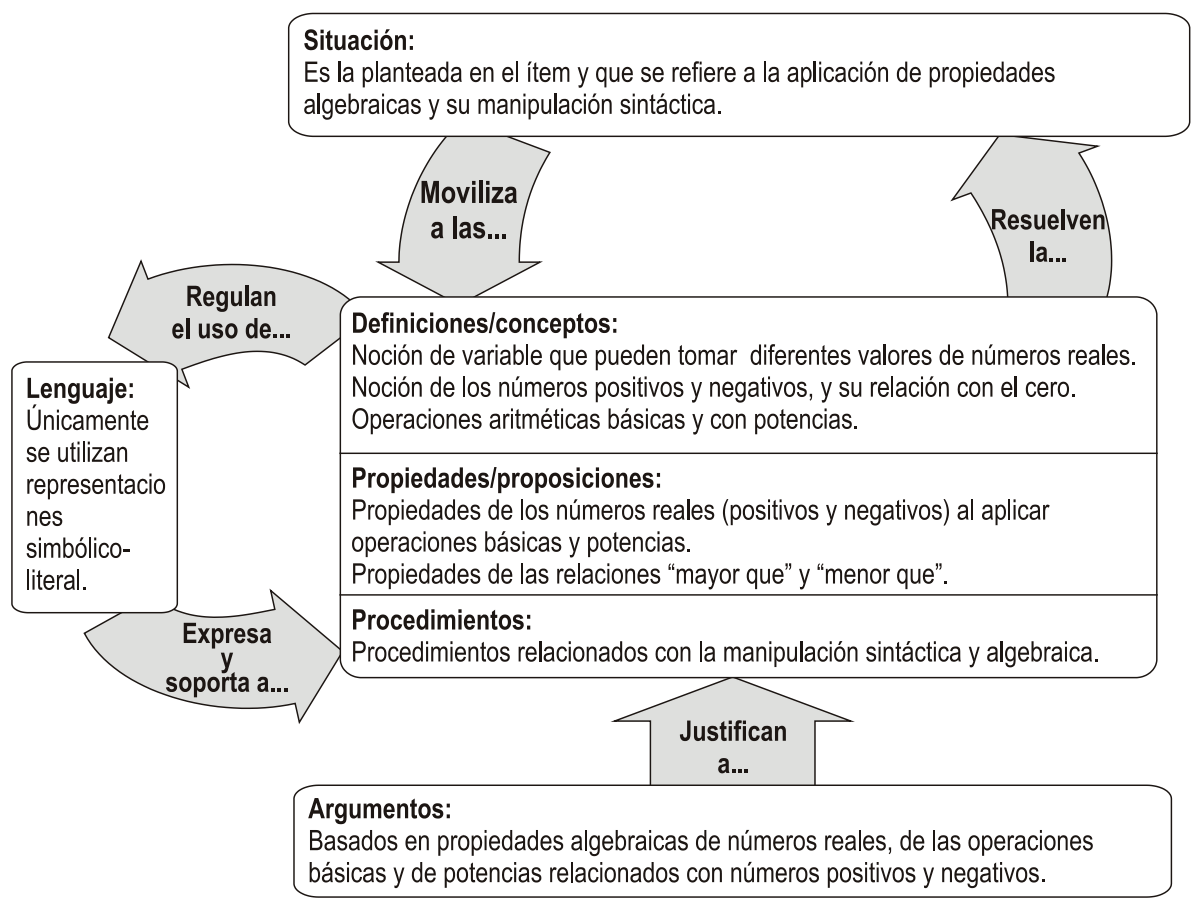

Ilustración 3. Configuración epistémica del ítem 3.

En este ítem se presentan supuestos generales que deben ser tomados en cuenta para que los participantes realicen un análisis se oriente a dos cosas principalmente: En primer lugar, el manejo -y posibles dificultades- de las propiedades de los números positivos y negativos al realizar operaciones básicas y con potencias que podría llevar a develar algunos conflictos semióticos presentes; por otro lado, el tipo de argumento que utilicen proporcionará información sobre su nivel de algebrización de los participantes por la manera en que se movilicen los objetos durante la práctica matemática. 


\section{ANÁLISIS Y DISCUSIÓN}

\section{Ítem 1: Conflictos semióticos}

En el primer ítem aparecieron varios conflictos semióticos cuando los participantes lo resolvieron. Muchos de éstos se pueden vincular con analogías o el uso de la intuición a partir de conocimientos previos. Los conflictos semióticos detectados con mayor incidencia aparecen a continuación:

Tabla 1.

\begin{tabular}{|c|l|c|}
\hline Núm. & \multicolumn{1}{|c|}{ Descripción } & $\begin{array}{c}\text { Porcentaje de } \\
\text { aparición }\end{array}$ \\
\hline 1 & $\begin{array}{l}\text { Se considera que la expresión } a^{2}=b \text { implica que } a=\sqrt{b} \text {, es decir, } \\
\text { no se considera la definición de valor absoluto o la posibilidad de } \\
\text { que la raíz cuadrada pueda ser negativa. }\end{array}$ & $27.7 \%$ \\
\hline 2 & $\begin{array}{l}\text { Se consideran erróneamente las operaciones inversas de las } \\
\text { operaciones: la inversa de la suma es la división }(a+b=c \text { implica } \\
\left.\text { que } a=\frac{b}{c}\right) \text {, la inversa de la multiplicación es la resta }(a b=c \\
\text { implica que } a=c-b) \text { o la inversa de la suma es la misma suma } \\
(a+b=c \text { implica que } a=b+c) .\end{array}$ & $23.1 \%$ \\
\hline 3 & $\begin{array}{l}\text { Se aceptan y utilizan expresiones como }(a+b)^{2}=a^{2}+b^{2}, \\
\sqrt{a^{2}+b^{2}}=a+b \text { o } a\left(\sqrt{b^{2}+c^{2}}\right)^{2}=a b+c \text { donde se aplica una } \\
\text { "distributividad" de la potencia (o raíz cuadrada) sobre la suma. }\end{array}$ & $20.5 \%$ \\
\hline 4 & $\begin{array}{l}\text { Se considera que } a=-b^{2} \text { implica que } \sqrt{a}=-b, \text { es decir, se omite el } \\
\text { signo “- en el proceso. }\end{array}$ & $10.8 \%$ \\
\hline 5 & $\begin{array}{l}\text { Consideran una simplificación errónea en la división: } \frac{a}{\frac{1}{b}}=\frac{b}{a} . \\
\text { distribuye sobre el producto: }(a b)^{2}=a^{2} b .\end{array}$ & $10.8 \%$ \\
\hline
\end{tabular}

Es interesante el uso de los números negativos. En el caso más recurrente de la tabla solo se considera que la raíz cuadrada de un número real puede tomar valores positivos. En este momento, hay que decirlo, el signo "-" no se utiliza como inverso aditivo o como una operación (resta), sino como indicativo de que se considera un valor menor a cero. Esto se puede constituir en una dificultad 
para el manejo adecuado del valor absoluto, pero también es una muestra más de que de manera automática no se consideran los negativos.

Además, esto se hizo patente en el cuarto caso de la tabla, donde casi el $11 \%$ de los participantes al momento de utilizar la raíz cuadrada, omiten el signo "-" en el proceso y lo consideran ajeno al mismo número.

Llama la atención que, a pesar de esa dificultad que representan los números negativos, o dicho de manera más apropiadamente, la interpretación del signo "-" como el inverso aditivo de un número, ocurrió que errores como $-\sqrt{a}=\sqrt{-a},-(a b)=(-a)(-b)$ y $-(a b)^{2}=\left(-a^{2}\right)\left(-b^{2}\right)$ aparecen relativamente poco: $2.6 \%$ de los participantes los cometieron.

Otro de los conflictos que se detectaron en casi una cuarta parte de los casos se reflejó en el uso erróneo de las operaciones inversas. Los casos comunes fueron considerar que, durante el despeje, la operación inversa de la suma es la división y que la operación inversa de la multiplicación es la resta. Aunque los alumnos pueden estar conscientes de la relación entre operaciones inversas, como menciona Kieran (2003), el cambio en el formato del algoritmo de las operaciones puede resultar confuso, pues se pasa de operaciones en dos o más renglones (que incluso permiten una verificación inmediata) a operaciones que se escriben en un solo renglón. Además, como ya se ha mencionado, se añade la dificultad de que en el contexto aritmético las operaciones producen una respuesta, mientras que en el algebraico no necesariamente es así. Todo ello promueve un conflicto en el significado de una operación inversa como proceso reversible de otra operación.

El tercer caso con más incidencias tiene que ver con una aplicación errónea de la distributividad de las potencias y raíces cuadradas sobre la suma. En este sentido Fischbein (1999) menciona que para los alumnos es una solución razonable desde la intuición a pesar de los posibles conflictos a los que se enfrenten. En buena medida esto se debe a que la multiplicación se explica en el nivel primaria como si fuese una suma abreviada, mientras que en el nivel secundaria, elevar a una potencia se explica como si fuese una forma de multiplicación abreviada y la raíz cuadrada como el proceso inverso de elevar al cuadrado. Al observar este proceso académico se hace "normal" que los participantes utilicen estas "propiedades" y, en el caso del cuadrado de la suma (o una factorización), omitan el término $2 a b$ que parece surgido de la nada. 


\section{Ítem 2: Tipos de estrategias y nivel de algebrización}

El segundo ítem considerado es, como ya se ha mencionado, una actividad de tipo generacional donde los participantes tendrían que interpretar un texto y resolver una situación que podría ser trabajada con el soporte de materiales concretos o de representaciones geométricas. En el contexto del objetivo de este trabajo, el ítem podía ser resuelto utilizando un sistema de ecuaciones o vía estrategias aritméticas.

Se tuvo que $82 \%$ de los participantes contestaron y solo $44 \%$ de ellos (36\% del total) contestaron correctamente, independientemente de la estrategia utilizada.

En general se identificaron tres tipos de estrategias:?

- Las aritméticas, que fueron aquellas en las que no aparecen representaciones algebraicas o, a lo mucho, no son útiles. Entre estas estregias se identificó el uso del conteo, que correspondió a representar y enumerar todos los elementos (los cubos) con diferentes acomodos, o bien probar casos particulares para irse acercando a la solución por ensayo y error.

- Las mixtas, que se refiere a estrategias que mezclaron una estrategia algebraica que fue abandonada para proseguir con una estrategia aritmética. Más adelante se amplía más al respecto.

- Las algebraicas, que fueron estrategias donde se utilizan, correcta o incorrectamente, expresiones y propiedades algebraicas que buscan resolver la situación planteada.

Al realizar el conteo de cada uno de los tipos de estrategia se obtuvieron los datos que se presentan en la tabla 2:

7 Se presentaron cinco casos (2.6\% del total) en el que se enuncia el resultado, pero sin procedimiento que permita determinar el tipo. Estos casos no están considerados en los datos. 
Tabla 2. Respuestas y estrategias presentes en el ítem $2^{8}$

\begin{tabular}{|cc|c|c|}
\cline { 3 - 4 } \multicolumn{1}{c|}{} & \multicolumn{2}{c|}{ Tipo de respuesta } \\
& Correcto & Incorrecto \\
\hline \multirow{3}{*}{ Tipo de estrategia } & $38(60 \%)$ & $25(40 \%)$ \\
\cline { 2 - 4 } & Mixto & $3(60 \%)$ & $2(40 \%)$ \\
\cline { 2 - 4 } & Algebraico & $25(29 \%)$ & $60(71 \%)$ \\
\hline
\end{tabular}

Se puede observar que en los dos primeros renglones hay más respuestas correctas (comparadas con las respuestas incorrectas) que en el último. Al aplicarse una prueba de independencia se obtuvo un valor $p$ de 0.0005 y ello nos lleva a rechazar una hipótesis de que existe una independencia entre el tipo de estrategia y el tipo de respuesta proporcionada. Es decir, pareciera que existe una relación entre que los participantes utilizaran un cierto tipo de estrategia (la aritmética) y la posibilidad de contestar correctamente. Esto deja el reto de que los alumnos construyan la conexión hacia lo algebraico y también "mantener viva la conexión álgebra hacia aritmética, es decir, desarrollar la habilidad de ir y venir entre los dos niveles de concepción y de ver las ventajas de ser capaz de escoger una perspectiva u otra, dependiendo del problema que se tenga que resolver" (Kieran, 1992, p. 411).

En los casos donde los participantes utilizaron estrategias mixtas se puede observar cómo se abandona la estrategia algebraica para recurrir al conteo o al tanteo (ver, por ejemplo, llustración 4).

$$
\begin{aligned}
& \text { Baltazar? } \\
& x^{2}-7=y^{2}+10 \\
& 81-7=74 \\
& x^{2}-y^{2}-7-10=0 \\
& 8 \times 8=64+10=74 \\
& x^{2}-y^{2}-17=0 \\
& x^{2}=y^{2}+r \\
& 74 \text { cubos } \\
& (a)(a)=18)(8)+7 \\
& \Rightarrow=\begin{array}{c}
6417 \\
81-81
\end{array}
\end{aligned}
$$

Ilustración 4. Estrategia mixta del participante g02-031 con respuesta correcta.

\footnotetext{
${ }^{8}$ Los porcentajes entre paréntesis corresponden al total por renglón, es decir, por tipo de estrategia.
} 
Se observa cómo se comienzan a utilizar expresiones algebraicas, aunque hay un momento en que se busca resolver la situación con algo que podría ser más conocido para los participantes y es consistente con el trabajo escolar de aritmética que se enfoca, por ejemplo, en obtener resultados más que representaciones de las relaciones planteadas en la situación (Kieran, 2003). Consideramos que en casos como estos, los participantes muestran, a lo más, un nivel 2 de algebrización ya que se utilizan representaciones simbólicas (literales) pero su manejo analítico es limitado por el uso de otro tipo de estrategias.

Algunos participantes mostraron estrategias aritméticas que sugieren niveles protoalgebraicos que incluso se pueden establecer como el nivel 0. Tal es el caso de estrategias como el conteo (llustración 5) y ensayo-error, donde los dibujos se utilizan como un apoyo para la estrategia usada y no para desarrollar razonamientos geométricos.

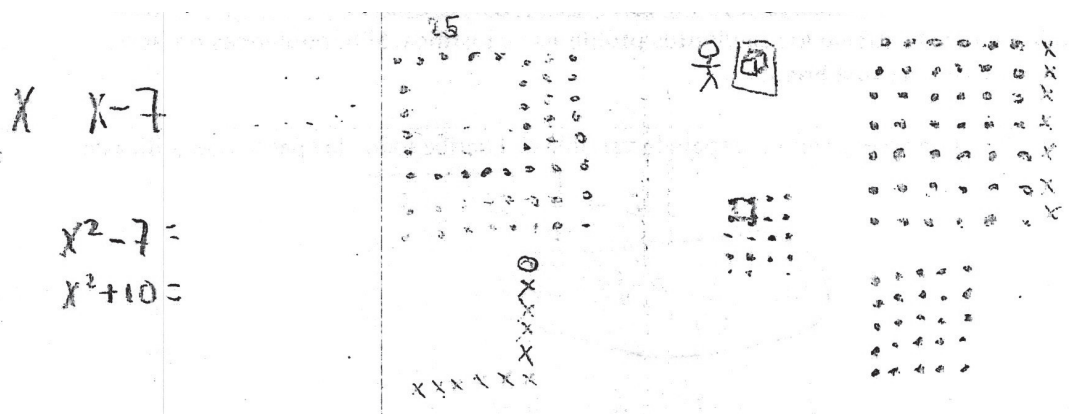

Ilustración 5. Respuesta del participante g04-008 con una estrategia de conteo (aritmética) y donde las expresiones algebraicas no son útiles.

$$
\begin{aligned}
& 4 x+7=1 \quad x=\frac{-6}{4} \\
& 4 x+4-10=1 \quad 4\left(-\frac{6}{4}\right)+4-10=1 \\
& -\frac{24}{4}+4-10=1 \\
& -6+4-10-6= \\
& -1+4= \\
& -13=0
\end{aligned}
$$

Ilustración 6. Respuesta del participante g07-021. 
Por otro lado, cuando los participantes utilizaron una estrategia algebraica cometieron errores en el planteamiento de las ecuaciones al no darle un sentido apropiado a la noción de variable, como es el caso del participante g07-021 (llustración 6) donde se maneja una expresión lineal (lo cual elimina la posibilidad de calcular áreas) y también se considera que la segunda variable no existe en sí, sino que es sustituida por un "1".

En un caso como este, se podría pensar en un nivel 3 de algebrización, sin embargo la falta de sentido para la segunda variable que se compensa con el uso de un número en particular, sugiere que el alumno puede llevar a cabo actividades del nivel 2 y podría estar en un proceso de transición.

Por otro lado, se puede mencionar que una vez que los participantes pudieron plantear expresiones algebraicas (correctas o incorrectas) se añadió la aparición de errores en la manipulación de las expresiones para resolverlas. En este segundo momento aparecieron nuevamente fenómenos como los mencionados en el ítem 1.

Asimismo hubo participantes que muestran un nivel 3 de algebrización al plantear expresiones algebraicas adecuadas (como un sistema de ecuaciones cuadráticas) y llevar a cabo las manipulaciones necesarias para obtener el resultado.

\section{Ítem 3: Conflictos semióticos y tipos de argumentaciones}

Como se menciona en la sección anterior, en este último ítem se pidió a los participantes que llevaran a cabo una actividad matemática global de meta-nivel que requiere la manipulación algebraica orientada a la identificación y movilización de propiedades de los números reales en términos de operaciones y números positivos y negativos.

Llama la atención de que, a diferencia del ítem anterior, para este la gran mayoría de los participantes lo intentaron: del 97\% de ellos, 74\% dio una respuesta final correcta, aunque en muchas ocasiones la conclusión se obtuvo con argumentos erróneos y ello se abordará un poco más adelante.

Una de las observaciones principales que saltaron a la vista es el conflicto semiótico que lleva comúnmente a los alumnos a confundir la noción de número negativo con el inverso aditivo. En otras palabras, cuando se utilizan variables

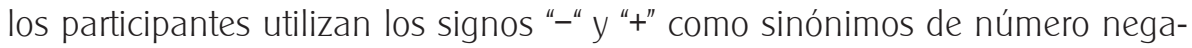
tivo y positivo, respectivamente (ver la llustración 7), o bien expresan la calidad de un número negativo (genérico) anteponiendo el signo "-“" como si fuese el inverso aditivo del número original (ver la llustración 8). 


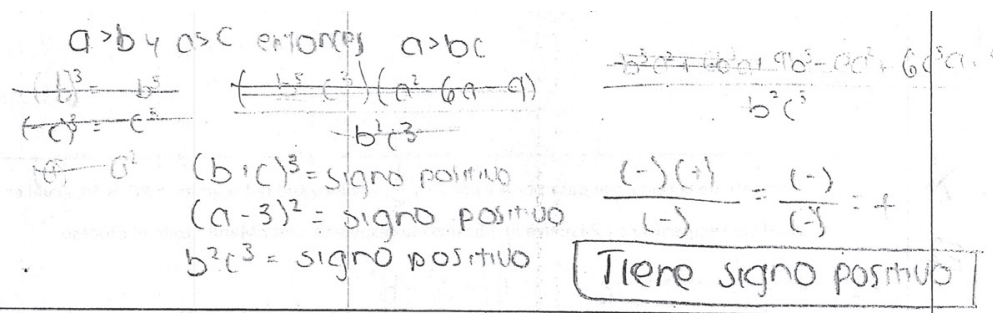

Ilustración 7. Respuesta del participante g04-005 donde se expresa lo positivo y lo negativo de un número con los símbolos "+" y "-". Esto se enfatiza con la respuesta final "Tiene signo positivo".

$$
\begin{aligned}
& \begin{array}{l}
\frac{(-b+c)^{3}(a-3)^{2}}{b^{2} c^{3}}=\frac{\left(-b^{3}-c^{3}\right)\left(a^{2}+9\right)}{\left(c b^{2} c^{3}\right)} \\
\approx \frac{-a b^{3}-a b^{3}-a^{2} c^{3}-9 c^{3}}{-\left(b^{2} c^{3}\right)} \leftarrow \text { negativo5> megativos) esos entre menes }
\end{array} \\
& \text { ha respuesto es positivo }
\end{aligned}
$$

Ilustración 8. Respuesta del participante g02-030 donde los números negativos se expresan como si fuesen inversos aditivos.

Además, en algunos casos este tipo de conflictos se vio mezclado con otros, como los detectados en el ítem 1 que muestran la falsa "distributividad" de la potencia sobre la suma y que se muestra en la llustración 9.

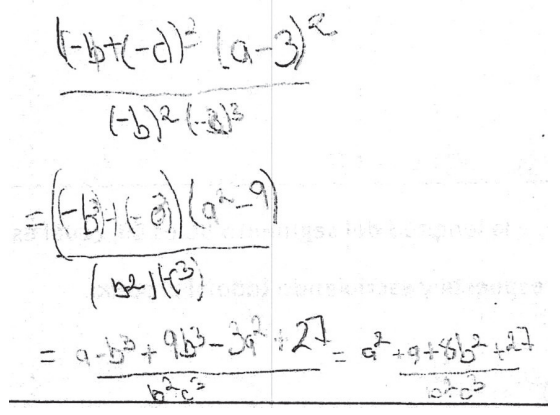

Ilustración 9. Respuesta del participante g03-009. A pesar de ello, el participante dio una respuesta correcta al final. 
Los tipos de argumentación que los participantes utilizaron para obtener las respuestas, se clasificaron tomando como una referencia las propuestas de Larios (Larios, 2018; Larios, Arellano y González, 2018), ya que este análisis en concreto ayuda a establecer una relación con los niveles de algebrización de los participantes en la aplicación (ver Tabla 3).

Tabla 3. Tipos de respuesta y propuesta niveles de algebrización en el ítem 3.

\begin{tabular}{|l|l|c|}
\hline \multicolumn{1}{|c|}{ Esquema de argumentación } & \multicolumn{1}{|c|}{$\begin{array}{c}\text { Nivel de } \\
\text { algebrización }\end{array}$} & Ocurrencia $^{9}$ \\
\hline $\begin{array}{l}\text { Empíricos. Se generaliza con uno o dos casos particu- } \\
\text { lares de números y las expresiones algebraicas están } \\
\text { ausentes o no tienen utilidad. }\end{array}$ & Niveles 0 y 1 & 60 (32\%) \\
\hline $\begin{array}{l}\text { Simbólicos. Hay un desarrollo simbólico (correcto o no) } \\
\text { que no considera propiedades y no se puede obtener } \\
\text { una conclusión. (Ver llustración 10.) }\end{array}$ & Niveles 1 y 2 & $64(34 \%)$ \\
\hline $\begin{array}{l}\text { Fácticos. La justificación es una serie de pasos realiza- } \\
\text { dos como si fuesen un algoritmo. (Ver llustración 11.) }\end{array}$ & Niveles 1 y 2 & $27(14 \%)^{10}$ \\
\hline $\begin{array}{l}\text { Analíticos. Se sigue una cadena deductiva de argu- } \\
\text { mentos que se basan en propiedades (de los números } \\
\text { y de las operaciones). }\end{array}$ & Nivel $36(19 \%)$ \\
\hline
\end{tabular}

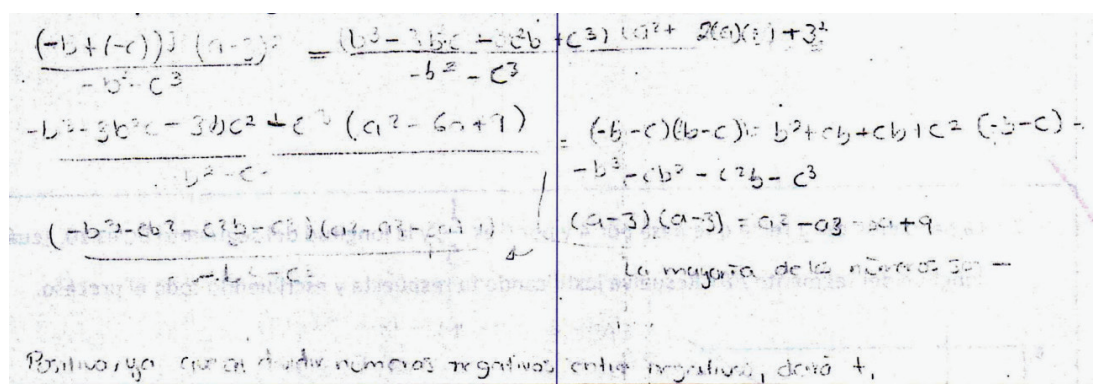

Ilustración 10. Respuesta del participante g01-014 de tipo simbólico.

\footnotetext{
${ }^{9}$ Los porcentajes corresponden a la proporción con respecto a las participantes que contestaron.

10 Se registraron tres casos en que aparecen respuestas que mezclan evidencia del uso de esquemas fácticos con los simbólicos y los empíricos.
} 
El desarrollo algebraico no es correcto y el conflicto semiótico en el uso del símbolo "-" lo lleva a una conclusión incorrecta con base a un conteo y una percepción visual.

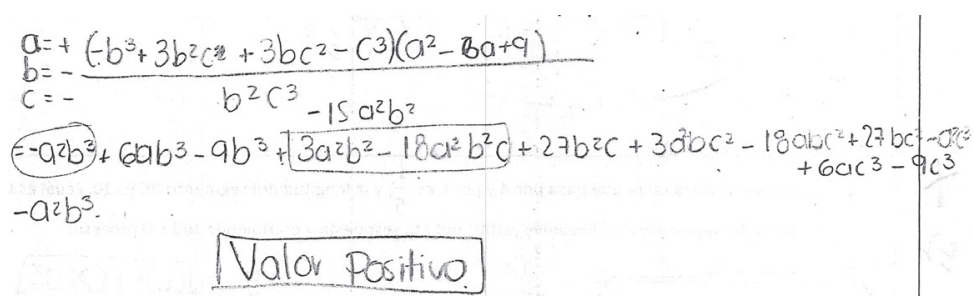

Ilustración 11. Respuesta del participante g06-021 de tipo fáctico.

Ahora bien, como las posibles respuestas del ítem solo eran dos ("positivo" y "negativo"), se consideró más importante observar el tipo de respuesta en términos del tipo de argumentación y si este fue correcto o no. En ocasiones, de hecho, los participantes dieron una respuesta correcta basándose en argumentos erróneos. El resumen de esto se presenta en la tabla 4.Tabla 4. Tipo de respuesta y tipo de justificación. ${ }^{11}$

\begin{tabular}{|l|c|c|}
\hline Esquema de argumentación & Justificación correcta & Justificación errónea \\
\hline Empíricos & $45(75 \%)$ & $15(25 \%)$ \\
\hline Simbólicos & $39(61 \%)$ & $25(39 \%)$ \\
\hline Fácticos & $2(7 \%)$ & $25(93 \%)$ \\
\hline Analíticos & $31(86 \%)$ & $5(14 \%)$ \\
\hline
\end{tabular}

Llama la atención que los argumentos de tipo empíricos pareciera que les dieron más control a los participantes (un tercio los utilizó), pero los principales errores provienen de una aplicación errónea de propiedades de operaciones (errores de copiado, triviales o del tipo mencionados en el ítem 1). Sin embargo, la aceptación de un caso como requisito suficiente para generalizar se mantuvo en esos casos.

11 Los porcentajes corresponden a las proporciones por renglón. 


\section{CONCLUSIONES}

A pesar de que los participantes ya habían concluido sus estudios de bachillerato, una proporción apreciable abordaron los ítems con estrategias de niveles protoalgebraicos. Esto muestra que las herramientas algebraicas no se han internalizado apropiadamente como para que se utilicen de manera eficiente. Si bien no es la única manera de resolver estos problemas, las estrategias algebraicas serán en los futuros alumnos de las carreras de ingeniería una herramienta potente para abordar situaciones, estudiar otros conceptos, lograr una optimización de modelos matemáticos, etcétera.

No obstante, y al igual que en los tipos de argumentos utilizados por los alumnos, pareciera que las estrategias y los argumentos más básicos, más conocidos y manejables (las aritméticas y los empíricos, respectivamente), proporcionan más control a los participantes para las respuestas y entonces "sacrifican" procesos y métodos más complejos y generalizados en aras de obtener una respuesta satisfactoria. Esto en realidad refuerza la idea de que se necesita dirigir esfuerzos en los niveles preuniversitarios para lograr que los alumnos le otorguen un sentido apropiado al álgebra. Procesos matemático-cognitivos como la generalización, la unitarización, formalización, ostensión y transformación (Godino et al., 2014) se presentan como puntos a desarrollar en propuestas didácticas en este sentido.

Además, y ligado precisamente a los significados construidos por los participantes con respecto a los objetos algebraicos, se añaden los conflictos que se han descrito, principalmente en los ítems 1 y 3 , los cuales se pueden convertir en obstáculos para los participantes en una posible carrera de ingeniería.

Llama la atención que algunos de estos conflictos están vinculados con objetos que los alumnos utilizan desde varios años antes en el contexto escolar, pero que no dejan de tener cierto grado de antiintuitivos. Como se mostró, la característica de un número de ser negativo es confundida en ocasiones con la noción de inverso aditivo y al parecer esto está vinculado con el hecho de que se utiliza el mismo signo ("-") para representarlas. Así, la ambigüedad en la notación parece ayudar a esa confusión. El trabajo didáctico debe entonces involucrar una reflexión por parte de los docentes sobre la diferencia entre ambos objetos (sus conceptos, propiedades y representaciones).

Una de las posibles implicaciones de lo anterior, está lo relacionado con la raíz cuadrada de un número real no negativo. Como se mencionó, se tiene una preferencia por considerar solo las posibilidades positivas de una raíz cuadrada 
(que, además, en muchas ocasiones no se le antepone un signo "+") e ignorar las opciones negativas (que requieren el uso del signo "-"). Esto podría tener un efecto contraproducente al estudiar el concepto de valor absoluto en los cursos de cálculo del primer año de la carrera.

Por otro lado, en los procesos matemáticos de los participantes se mostró que la relación entre las operaciones inversas, como procesos reversibles en una relación de equivalencia, no está clara. Es posible que esta situación tenga su origen en la educación básica, donde se presentan las cuatro operaciones básicas que, si bien están relacionadas entre sí, operan de diferente manera: La suma añade, la resta quita, la multiplicación es una suma abreviada y la división es una repartición. Sin embargo, hay que mencionar que esta estrategia en los primeros años de la vida escolar está orientada por el desarrollo cognitivo del alumno en ese momento, aunque se puede constituir en un obstáculo con el paso del tiempo. Esto muy bien podría ser un ejemplo de la paradoja cognitiva de las matemáticas (Duval, 2010) ya que en su momento los niños tienen que utilizar diferentes representaciones de las operaciones aritméticas para entenderlas, pero esas representaciones tienen limitaciones inherentes que deben ser superadas cuando se comprenden las operaciones mismas. Es por ello que es necesario que los profesores de todos los niveles proporcionen a sus alumnos situaciones, en las que se utilicen diferentes representaciones y procedimientos que sean representativos.

Como se mencionó al inicio de este trabajo, aquellos participantes que ingresen a la Facultad tomarán durante su primer año cursos de cálculo diferencial y álgebra lineal. Si bien en el primero de esos cursos se puede hacer una aproximación basada en nociones de movimiento y aplicaciones (Arcos, 2004; Pino-Fan et al., 2011), en el de álgebra lineal se abordan temas que tienden al manejo de conceptos y estructuras algebraicos con la finalidad de que sirvan como base y aplicación a otros cursos de matemáticas y de física. Estos cursos requieren al menos un nivel 3 de algebrización para un avance exitoso e, incluso, se tocan aspectos que puedan requerir un nivel mayor, pues "el uso de estrategias algebraicas para el planteamiento y la resolución de situaciones matemáticas (puras y aplicadas) ayuda para comprender los conceptos más complejos vistos en los cursos de Ingeniería" (Cuevas et al., 2018, p. 167). 


\section{REFERENCIAS}

Arcos Q., J. I. (2004). Rigor o entendimiento, un viejo dilema en la enseñanza de las matemáticas: El caso del cálculo infinitesimal. Tiempo de Educar, 5(10), 77-110.

Aznar, A., Baccelli, S., Figueroa, S., Distéfano, M. L. y Anchorena, S. (2016). Las funciones semióticas como instrumento de diagnóstico y abordaje de errores. Bolema. Boletim de Educação Matemática, 30(55), 670-690. https://doi.org/10.1590/19804415v30n55a18.

Cuevas S., O., Larios O., V., Peralta G., J. X. y Jiménez S., A. R. (2018). Mathematical knowledge of students who aspire to enroll in engineering programs. International Electronic Journal of Mathematics Education, 13(3), 161-169.

Duval, R. (2010). Sémiosis, pensée humaine et activité mathématique. Amazônia - Revista de Educação em Ciências e Matemáticas, 6(11), 126-143.

Eco, U. (2005). Tratado de semiótica general. Random House Mondadori.

Fischbein, E. (1999). Conocimiento intuitivo y conocimiento lógico en la actividad matemática. Pitagora Editrice Bologna y Grupo Editorial Iberoamérica.

Frost, J. (2015). Disappearing x: When solving does not mean finding the solution set. Journal of Mathematical Behavior, 37, 1-17.

Godino, J. D. y Batanero B., C. (2003). Semiotic functions in teaching and learning mathematics. En M. Anderson, A. Sáenz-Ludlow, S. Zellweger y V. V. Cifarelli (edits.), Educational perspectives on mathematics as semiosis: From thinking to interpreting to knowing (pp. 149-167). Legas Publishing.

Godino, J. D., Aké T., L. P., Gonzato, M. y Wilhelmi, M. R. (2014). Niveles de algebrización de la actividad matemática escolar. Implicaciones para la formación de maestros. Enseñanza de las Ciencias, 32(1), 199-219. https://doi.org/10.5565/rev/ensciencias.965.

Godino, J. D., Batanero B., C. y Font Moll, V. (2007). The ontosemiotic approach to research in mathematics education. ZDM. The International Journal on Mathematics Education, 39(1-2), 127-135.

Godino, J. D., Neto, T., Wilhelmi, M. R., Aké T., L. P., Etchegaray, S. y Lasa, A. (2015). Niveles de algebrización de las prácticas matemáticas escolares. Articulación de las perspectivas ontosemiótica y antropológica. AIEM, 8, 117-142.

Hjelmslev, L. (1971). Prolegómenos a una teoría del lenguaje. Editorial Gredos.

Kieran, C. (1992). The learning and teaching of school algebra. En D. A. Grouws (edit.), Handbook of research on mathematics teaching and learning (pp. 390-419). Macmillan.

Kieran, C. (2003). The transition from arithmetic to algebra: A model for conceptualizing school algebra and the role of computer technologyin supporting the development 
of algebraic thinking. En E. Filloy Y. (edit.), Matemática educativa. Aspectos de la investigación actual (pp. 121-142). Cinvestav y F.C.E.

Larios O., V. (2018). Un estudio exploratorio de los esquemas que emplean los alumnos de bachillerato para validar resultados matemáticos. Transformación, 14(2), 190-201.

Larios O., V., Arellano C., C. y González G., N. (2018). Análisis de argumentos producidos por alumnos de bachillerato al resolver problemas de geometría. Redimat. Journal of Research in Mathematics Education, 7(3), 280-310

Larios O., V., Valerio L., T. J., Ochoa C., R., Spíndola Y., P. I., Sosa G., C. y Fajardo A., M. C. (2017). Dificultades en el aprendizaje del Álgebra de bachillerato: Un estudio exploratorio. Pädi. Revista de Didáctica de las Ciencias y la Ingeniería, 1(1), 53-71.

Larrazolo, N., Backhoff, E. y Tirado, F. (2013). Habilidades de razonamiento matemático de estudiantes de educación media superior en México. Revista Mexicana de Investigación Educativa, 18(59), 1137-1163.

Martínez H., A., Rojas R., A. L. y Villanueva G., C. E. (2015). Actitud del docente: una visión del alumno. En Memorias del $11^{\circ}$ Congreso Internacional de Ingeniería. Editorial Universitaria UAQ.

National Mathematics Advisory Panel [NMAP]. (2008). The Final Report of the National Mathematics Advisory Panel. U.S. Department of Education.

Pino-Fan, L. R., Godino, J. D. y Font M., V. (2011). Faceta epistémica del conocimiento didáctico-matemático sobre la derivada. Educaçāo Matematica Pesquisa, 13(1), 141-178.

Rojano C., M. T. (2006). Adquisición del lenguaje algebraico: El programa de investigación. En E. Filloy Y. (edit.), Matemática educativa, treinta años: Una mirada fugaz, una mirada externa y comprensiva, una mirada actual (pp. 55-67). Santillana y Cinvestav.

\section{VÍCTOR LARIOS OSORIO}

Dirección: Av. Miguel Hidalgo y Costilla s/n, Cerro de las Campanas, CP 76010, Querétaro, Qro., México vil@uaq.mx

Teléfono: $\quad$ +52 (442) 192-1200 ext. 7018 